Communications in Physics, Vol.21, No. 2 (2011), pp. 105-115

\title{
RADIO DETECTION OF THE SUN
}

\author{
PHAM TUAN ANH, DANG QUANG THIEU, PHAM NGOC DIEP, \\ PHAM NGOC DONG, NGUYEN VAN HIEP, DO THI HOAI, \\ PHAM THI TUYET NHUNG, NGUYEN THI THAO, \\ AND PIERRE DARRIULAT \\ VATLY, Institute for Nuclear Science and Technology
}

\begin{abstract}
The radio emission of the Sun has been measured using a radio interferometer including two Yagi antennas operated at $610 \mathrm{MHz}$. We report the observation of interferences and comment on the results.
\end{abstract}

\section{INTRODUCTION}

Radio astronomy was born less than a century ago and has since developed into one of the most successful branches of modern astrophysics, contributing major data to nearly all of its chapters, including distant galaxies, pulsars, active galactic nuclei, quasars, supernova remnants, molecular clouds and envelopes of giant stars. While, apart from a narrow window in the visible, higher frequency electromagnetic radiations are absorbed by the Earth atmosphere, radio waves reach the Earth essentially undisturbed and radio astronomy observations are made from ground. At variance with optical telescopes, radio telescopes can be operated day and night and under any weather condition. In tropical countries such as Vietnam, where the sky is very often obscured by fog or clouds, making useful optical observations nearly impossible, radio astronomy is therefore the only practical opportunity for domestic research in astrophysics.

Normal main sequence stars, such as our Sun, are very faint radio emitters and the radio sky does not show the stars which have been observed for several millennia by the naked eye and, more recently, by powerful telescopes. There is however an exception, a single exception, to this statement, which is precisely our Sun. The Sun is so close to the Earth that, in spite of its very faint radio emission, it still is the strongest radio source in our sky, at least hundred times stronger than major radio sources such as Cas A (a supernova remnant), Sgr A (the black hole at the centre of the Milky Way) or Cen A (the closest AGN, at the centre of two colliding galaxies). It can be observed with relatively modest instruments and such an observation is the subject of the present article.

In 1995, a solar eclipse occurred in South Viet Nam, in the province of Phan Thiet. On that occasion Prof. Nguyen Quang Rieu, an astronomer at the Meudon Observatory, near Paris, brought there a small radio interferometer that had been built by radio astronomers in Nancay (France) [1] and observed the decline, extinction and revival of the solar signal [2]. The interferometer was left in Vietnam as a gift in the hope that it could 
be of help for introducing modern astrophysics to Vietnamese students. Unfortunately, there being no teaching of modern astrophysics in Vietnam, it had been left dormant for nearly fifteen years. The work presented here reports on the refurbishing of this instrument and on observations of the Sun that have been made using it.

\section{PRINCIPLE OF THE METHOD}

Consider two antennas $A_{1}$ and $A_{2}$ separated by a distance $L=\left|\mathbf{A}_{\mathbf{1}} \mathbf{A}_{\mathbf{2}}\right|$ (baseline) and oriented in the Sun direction defined by unit vector $\mathbf{u}$ (Fig. 1). The radio signal emitted by the Sun covers a very broad frequency domain and the antennas collect whatever fraction of it falls within their bandwidth centred on frequency $f$. As a function of time $t$, the output voltage in antenna $A_{i}$ is $V_{i}=E_{i} \sin \left(2 \pi f t+\varphi_{i}\right)$. We may take $\varphi_{1}=-\varphi_{2}=1 / 2 \Delta \varphi$ by properly choosing the origin of time. The time delay between the signals received by the two antennas (the Sun is at infinity) corresponds to a phase difference $\Delta \varphi=2 \pi f \tau$ where $\tau=\mathbf{A}_{\mathbf{1}} \mathbf{A}_{\mathbf{2}} \cdot \mathbf{u} / c$.

Adding both signals, and writing $S=\exp (2 i \pi f t)\left\{E_{1} \exp (2 i \pi f \tau / 2)+E_{2} \exp (-2 i \pi f \tau / 2)\right\}$,
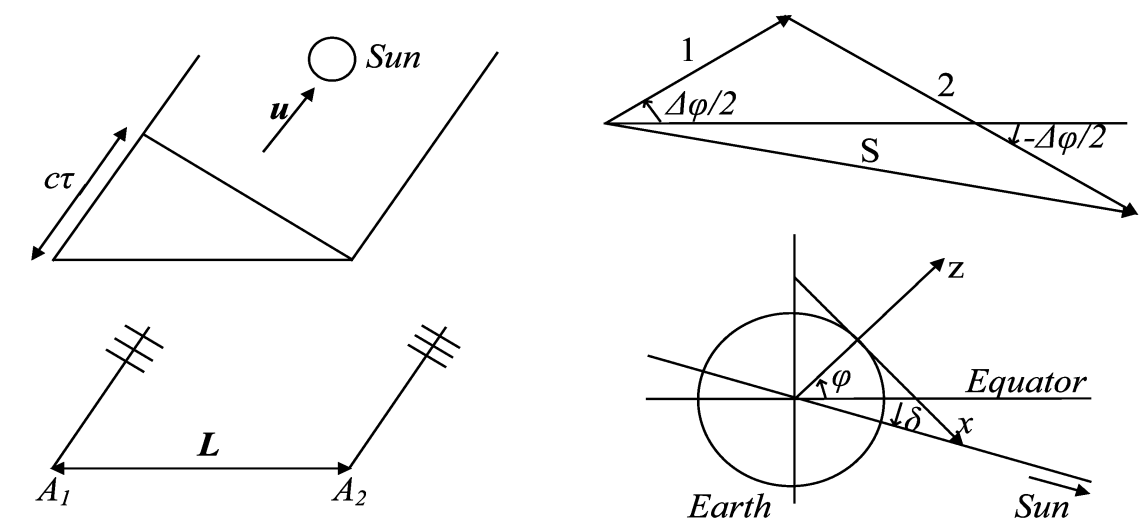

Fig. 1. Left: a two-antennas interferometer. Right up: adding the signals in the complex plane. Right down: Noon geometry.

the sum signal is the imaginary part of $S$. The first exponential oscillates very rapidly and averages to zero; its amplitude is the absolute value of the expression in the curly bracket which oscillates slowly with frequency $1 / \tau$. It reads: $S=\mid E_{1} \exp (2 i \pi f \tau / 2)+$ $E_{2} \exp (-2 i \pi f \tau / 2) \mid=\left\{E_{1}^{2}+E_{2}^{2}+2 E_{1} E_{2} \cos 2 \pi f \tau\right\}^{1 / 2}$. Writing the wave length $\lambda=c / f$ and the relative amplitude $r=E_{2} / E_{1}$, one may rewrite

$$
S=E_{1}\left\{1+r^{2}+2 r \cos \left(2 \pi \mathbf{A}_{\mathbf{1}} \mathbf{A}_{\mathbf{2}} \cdot \mathbf{u} / \lambda\right)\right\}^{1 / 2} .
$$

In order to evaluate the scalar product, we take a local reference frame having the $x$ axis horizontal toward south, the $y$ axis horizontal toward east and the $z$ axis vertical upward. As the baseline is approximately east-west, we write $\mathbf{A}_{\mathbf{1}} \mathbf{A}_{\mathbf{2}}=(\xi,-L+\eta, \zeta)$ where $\xi, \eta$, and $\zeta$ account for small deviations of the baseline from its nominal value. The unit 
vector $\mathbf{u}$ is a function of the latitude $\varphi$ of the place of observation, the declination $\delta$ of the Sun and the hour angle $H$ :

$\mathbf{u}=(-\cos \varphi \sin \delta+\sin \varphi \cos \delta \cos H,-\cos \delta \sin H, \sin \varphi \sin \delta+\cos \varphi \cos \delta \cos H)$.

At noon, when $H=0, \mathbf{u}=(\sin [\varphi-\delta], 0, \cos [\varphi-\delta])$.

Finally, the modulus of the added signal reads:

$$
S=E_{1}\left\{1+r^{2}+2 r \cos (2 \pi D / \lambda)\right\}^{1 / 2}
$$

with:

$$
\begin{aligned}
D= & \mathbf{A}_{\mathbf{1}} \mathbf{A}_{\mathbf{2}} \cdot \mathbf{u} \\
= & \xi(-\cos \varphi \sin \delta+\sin \varphi \cos \delta \cos H) \\
& +(L-\eta) \cos \delta \sin H+\zeta(\sin \varphi \sin \delta+\cos \varphi \cos \delta \cos H)
\end{aligned}
$$

For $r=1$ and $\xi=\eta=\zeta=0, S=\sqrt{2} E_{1}\{1+\cos (2 \pi D / \lambda)\}^{1 / 2}=2 E_{1}|\cos \pi D / \lambda|$ and $D=L \cos \delta \sin H$.

At equinox $(\delta=0)$ around noon, in the approximation where $\lambda \ll L$, the period of the interference is $T \sim \lambda / L$. In minutes, $T \sim(720 / \pi) \lambda / L$; for $\lambda=50 \mathrm{~cm}$ and $L=25 \mathrm{~m}$, $T \sim(720 / \pi) / 50=4.6 \mathrm{mn}$.

The antennas have a lobe width such that the Sun stays within their range long enough to allow for the observation of several interference fringes without changing their orientation. Note that if the antennas are not perfectly pointing to the Sun, $D$, and therefore the phase and frequency of the interference term, are unaffected: all what may change are the absolute intensity and the ratio $r$. In practice, the Sun emission will be superimposed on a background that depends on the environment, which must be chosen sufficiently quiet electromagnetically for the background to vary slowly, or better to remain constant during the measurement.

The baseline must be long enough to allow for the observation of several interference oscillations. But the time it takes to scan one interference period should not be too short in order to have time to collect sufficient statistics and not to suffer from the apparent diameter of the Sun $(\sim 2 \mathrm{mn})$. A value of 13 fringes per hour, given above as an example, is a reasonable compromise.

\section{DESCRIPTION OF THE INTERFEROMETER}

The interferometer includes two Yagi antennas operated around $610 \mathrm{MHz}$, the signals of which are preamplified, added, amplified and shifted to $30 \mathrm{MHz}$ by standard super heterodyne. A general schematic of the apparatus is shown in Figure 2. The $30 \mathrm{MHz}$ signal is then sent to the detector, the amplified output DC voltage of which is continuously recorded in a PC. On Earth, we receive $\sim 3.10^{-21} \mathrm{~W} / \mathrm{m}^{2} / \mathrm{Hz}$ from the quiet Sun [3], equally shared between left and right polarizations. Therefore, with $1 \mathrm{MHz}$ bandwidth and $1 \mathrm{~m}^{2}$ effective antenna area, corresponding to an antenna temperature of the order $40 \mathrm{~K}$, we receive $\sim 10^{-15} \mathrm{~W}$ which need to be amplified to $\sim 100 \mathrm{nW}(\sim 3 \mathrm{mV}$ under $50 \Omega)$ implying a gain of $\sim 80 \mathrm{~dB}$ in power or $\sim 40 \mathrm{~dB}$ in amplitude. 




Fig. 2. Logic diagram of the electronics showing the antennas (Ant), preamplifiers (P), filter, super heterodyne (Oscillator-Mixer), amplifiers (A), variable attenuator, detector and counter. Circles with arrows indicate adjustments (VCO frequency, A2 gain, attenuation and detector range).

In the frequency range used here $(\sim 610 \mathrm{MHz})$ the main contributions to the background are from harmonics of TV emissions and mobile communication (GSM). In practice, it has proven impossible to operate in town from the roof of the laboratory, several strong emitters installed nearby preventing stable operation at the desired frequency. The measurements reported here were made in the surroundings of Hanoi, near Hoa Binh, in a place where electromagnetic pollution is low.

\section{Antennas}

We use standard TV antennas (YAGI type, channels 31 to 49,550 to $750 \mathrm{MHz}$ ) consisting of a folded dipole with 2 reflecting bars behind and 9 directing bars in front. As we did not measure their detailed characteristics, we can only quote their nominal gain, 15 $\mathrm{dB}$. This means that the power collected in the direction to which they point is the power collected by an effective area $A$ which is $G=10^{1.5}=31.6$ times higher than what an ideal isotropic antenna would collect, namely $\lambda^{2} / 4 \pi=0.020 \mathrm{~m}^{2}$. Their nominal effective area is therefore $A=0.63 \mathrm{~m}^{2}$ and the beam solid angle is $\Omega_{A}=4 \pi / G=0.40 \mathrm{sr}$ corresponding to a cone of $39^{\circ}$ half aperture.

They are installed on stable supports with equatorial mounting.

\section{Preamplifiers}

Each antenna is connected via a short $50 \Omega$ coaxial cable to a low noise preamplifier having a nominal gain of $20 \mathrm{~dB}$ for frequencies between 450 and $900 \mathrm{MHz}$ (TV band IV). Here, and in what follows, gains are referred to power, $20 \mathrm{~dB}$ corresponding to a factor 10 in amplitude. The preamplifiers are supplied with $\pm 15 \mathrm{~V} \mathrm{DC}$ via the very same coaxial cables used to transmit the signals by using "polarization T's" that allow for separating DC supply and RF signals. Their gains have been measured in the laboratory using a sine wave generator ${ }^{1}$ and a spectrum analyzer ${ }^{2}$ and found to differ by $1 \mathrm{~dB}$, meaning $r=1.12$ in Relation 1. As the effect on the interference is very modest, no attempt was made at adjusting the preamplifier gains.

The preamplifiers are connected via $25 \mathrm{~m}$ long $50 \Omega$ coaxial cables (attenuation $\sim 4 \mathrm{~dB}$ ) to an adder that is immediately followed by a filter, the response of which has

\footnotetext{
${ }^{1}$ Keithley 2920 RF Signal.

${ }^{2}$ Anritsu MS2663C.
} 
been measured in the laboratory. It is centred on $611 \mathrm{MHz}$ with a bandwidth of $\sim 15 \mathrm{MHz}$ at $3 \mathrm{~dB}$ and attenuates the signal by $6 \mathrm{~dB}$. It eliminates undesired signals at an early stage.

\section{Local oscillator and Mixer}

The mixer causes the filtered sum signal, with frequency $f_{R F}$, to beat with a fixed frequency wave in order to work at lower frequencies where working conditions are much easier. The fixed frequency wave is produced by a voltage controlled oscillator (VCO) with frequency $f_{C O}$ adjustable via a potentiometer. The output frequency $f_{R F}$ is such that $f_{C O}=f_{R F}-f_{I F}$. The choice of $30 \mathrm{MHz}$ for $f_{I F}$ is made by a filter at the output stage of the mixer, with a bandwidth of $\sim 6 \mathrm{MHz}$. In quiet conditions, one can tune $f_{C O}$ and select this way $f_{R F}$ at will around $610 \mathrm{MHz}$ within the $\pm 7.5 \mathrm{MHz}$ acceptance of the input filter. However, in cases where a strong signal exists at nearby frequencies, as is the case in the environment of our laboratory, it acts on $V_{C O}$ in such a way that the desired frequency cannot be maintained. During measurement, the frequency was continuously monitored using a frequency meter ${ }^{3}$. The dependence of $f_{C O}$ on $f_{R F}$ has been measured in the laboratory and found well behaved.

\section{$30 \mathrm{MHz}$ amplifiers and detector}

Two $30 \mathrm{MHz}$ amplifiers, $A 1$ and $A 2$, are used after the mixer. Both have a broad band width. $A 1$ has a fixed gain of $43 \mathrm{~dB}$ and the adjustable gain of $A 2$ was set at 20 $\mathrm{dB}$ in the present set of measurements. Gains and linearities have been measured in the laboratory. An adjustable precision attenuator, set at $3 \mathrm{~dB}$ during normal measurements, was inserted between $A 2$ and the detector to allow for an easy calibration of the latter. The detector includes a third amplifier at its input stage followed by a filter, a backward diode and an operational amplifier giving a DC output of up to $8 \mathrm{~V}$. The response, illustrated in Figure 3, is linear over a small adjustable $\sim 3 \mathrm{~dB}$ range with a full dynamic range of $\sim 20 \mathrm{~dB}$. The band width, measured between 29.9 and $30.6 \mathrm{MHz}$ at $3 \mathrm{~dB}$, is narrower than that of other units and defines therefore the overall bandwidth.

Table 1 summarizes the gains and bandwidths of the various components of the system.

\section{Data acquisition}

The detector DC output is converted to frequency and scaled in a $10 \mathrm{kHz}$ scaler which is read out every millisecond into a PC, its content being added to a memory which is transferred to the monitoring programme and cleared every second under LabView, providing both storage of the recorded data and online display of the time dependence.

\section{OBSERVATION OF INTERFERENCES}

Preliminary measurements have been made in various places in Ha Noi. They have shown the need to work in an electromagnetically quiet place and have given the

\footnotetext{
${ }^{3}$ LEADER LDC-824 $520 \mathrm{MHz}$ frequency meter.
} 
Table 1. Summary of gains and bandwidths

\begin{tabular}{ccc}
\hline \hline Component & Gain (dB in power) & Bandwidth \\
\hline Antennas + cables & $15-4=11$ & broad \\
\hline Preamplifiers & $20 / 21$ & broad \\
\hline Filter & -6 & $15 \mathrm{MHz}$ \\
\hline Oscillator/Mixer & -9 & $6 \mathrm{MHz}$ \\
\hline Amplifier A1 & 43 & broad \\
\hline Amplifier A2 & 20 & broad \\
\hline Attenuator & -3 & broad \\
\hline Detector & See Figure 3$)$ & 29.9 to $30.6 \mathrm{MHz}$ \\
\hline Global & 76 & $0.7 \mathrm{MHz}$ \\
\hline \hline
\end{tabular}

opportunity to upgrade the detector by adding new components and replacing or fixing old ones. The data presented here were collected in a single day, on June $25^{\text {th }} 2010$, near Hoa Binh.

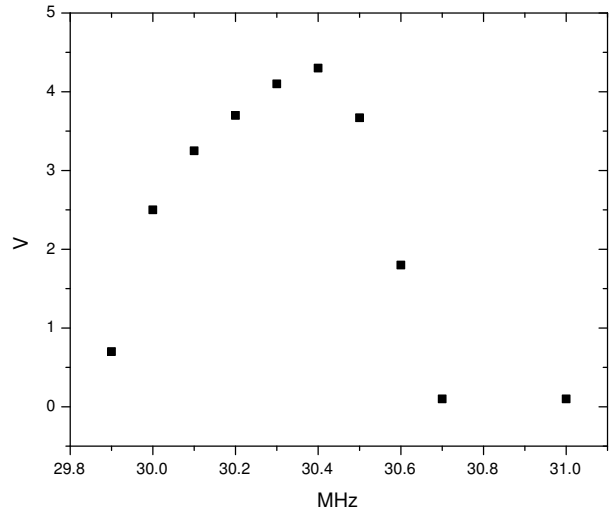

(a)



(b)

Fig. 3. Response of the detector. (a): lab measurement of the bandwidth for -37.6 $\mathrm{dBm}$ input corresponding to an amplitude of $4.17 \mathrm{mV}$. The detector $\mathrm{DC}$ output is shown in ordinate ( $4 \mathrm{~V}$ correspond to $20^{\prime} 000$ counts). (b): in situ measurement of the linearity of the detector around its working point; the number of counts recorded per second (in ordinate in units of 1000 counts) was measured in the field by changing the setting of the variable attenuator at detector input; the input amplitude, relative to the working point ( $3 \mathrm{~dB}$ attenuation, 22'900 counts per second), is shown in abscissa; the curve is the result of a quadratic fit. The detector potentiometer setting was $30.3 \mathrm{k} \Omega$ for both measurements. 
Two baselines were used, approximately east-west, with lengths of $22.9 \pm 0.1 \mathrm{~m}$ (runs 1 to 4 ) and $17.9 \pm 0.1 \mathrm{~m}$ (runs 5 to 15 ) respectively. In both cases the angle with the east-west direction was $4.4 \pm 0.7^{\circ}$ toward south-east/north-west. The configuration of the ground did not allow for an exactly horizontal baseline: the altitude of the western antenna was higher than the altitude of the eastern antenna by $1.56 \pm 0.10 \mathrm{~m}$ for the 22.9 $\mathrm{m}$ baseline and $1.44 \pm 0.10 \mathrm{~m}$ for the $17.9 \mathrm{~m}$ baseline. Data were collected between $\sim 10: 30$ am and $\sim 3: 45 \mathrm{pm}$ in 15 runs of $\sim 1000 \mathrm{~s}$ each. Time and VCO frequency were recorded at the start and at the end of each run.

Shortly after $10 \mathrm{am}$, with the antennas vertical, the background level was measured and the amplifier, attenuator and detector settings were tuned in such a way as to operate in the middle of the detector linear range, around 22'000 counts. A scan of VCO frequencies did not show any significant frequency dependence of the background level and the system was operated with VCO frequencies between 582.6 and $583.2 \mathrm{MHz}$. The linearity of the response was measured between $-3 \mathrm{~dB}$ and $+3 \mathrm{~dB}$ from the working point with the result that 194 counts correspond to a 1\% increase in amplitude (Figure 3 right). The measurement made in the laboratory using a sine wave generator set at $30.4 \mathrm{MHz}$ frequency (Figure 3 left) gives a calibration of the detector, $4.3 \mathrm{~V} \mathrm{DC}$ out (21'500 counts) for 4.17 $\mathrm{mV}$ in. Averaging over frequency for a uniform frequency distribution at the input of the detector between $29.9 \mathrm{MHz}$ and $30.6 \mathrm{MHz}$, namely a $0.7 \mathrm{MHz}$ interval, Figure $3 \mathrm{left}$ gives 15 '000 counts for the same $4.17 \mathrm{mV}$ in. Hence, on the working point (22'000 counts meaning $6.12 \mathrm{mV}$ at detector input) 194 counts correspond to $61 \mu \mathrm{V}$ giving an effective conversion factor of $3.2 \pm 0.3$ counts per $\mu \mathrm{V}$, the uncertainty being estimated to be of the order of $10 \%$.

The time dependence of the detected signal is shown in Figure 4 for two representative runs, with zero suppressed by 21'500 counts. The run in the left panel displays particularly quiet conditions while the run in the right panel shows evidence for non-linear background conditions. The amplitude of the solar interference is of the order of \pm 150 counts, slightly below one percent of the background level. Background fluctuations are usually slow and it was found that using a quadratic time dependence over each particular
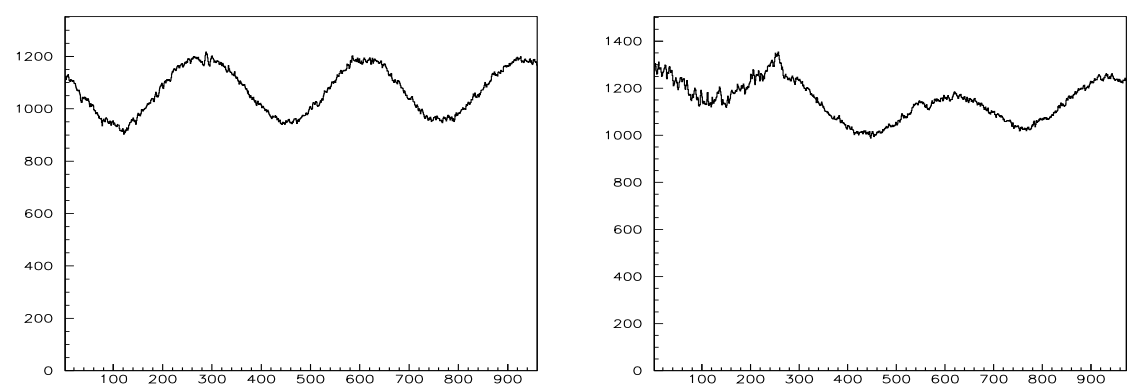

Fig. 4. Two representative runs; the time, in seconds, is shown in abscissa; the number of counts per second, after subtraction of $21^{\prime} 500$ counts, is shown in ordinate. 




Fig. 5. Slowly varying background obtained from preliminary linear background fits. The levels at the beginning (squares) and at the end (circles) of each run are shown together with the result of a quadratic fit (curve). Time, measured from noon in minutes, is shown in abscissa. Counts per second are shown in ordinate after subtraction of $21^{\prime} 500$ counts.

run was good enough an approximation. Only in one case has it been necessary to disregard a spike that lasted over 40 seconds. The data in the spike range have been replaced by a linear interpolation between data before and after the disturbance. However, small spikes such as that visible on the right panel of Figure 4 between 250 and $300 \mathrm{~s}$ have been kept.

The voltage oscillations measured in the antennas are the superposition of RF electric field oscillations having as respective sources the Sun and backgrounds of various origins. Within the bandwidth of the system they add up incoherently - their respective phases being unrelated - and the resulting amplitude of the RF signal is therefore obtained by adding the Sun and background signals in quadrature:

$$
\begin{aligned}
S_{\text {det }} & =\sqrt{\left(S_{\text {Sun }}^{2}+S_{\text {bg }}^{2}\right)} \\
& =\left(E_{1}^{2}\left\{1+r^{2}+2 r \cos (2 \pi D / \lambda)\right\}+B^{2}\right)^{1 / 2} \\
& \sim B+\left(E_{1}^{2} / B\right)\left\{1+r^{2}+2 r \cos (2 \pi D / \lambda)\right\} / 2
\end{aligned}
$$

where $B$ is the amplitude of the background and where the second expression applies when $E_{1}^{2} / B^{2} \ll 1$. It is therefore $E_{1}^{2} / B^{2}$ which is at the percent level, implying that $E_{1} / B$ is at the $10 \%$ level. By squaring each side of the above relation, one obtains an equivalent relation expressing the equality of powers. Radio astronomers usually prefer to use this latter form. 

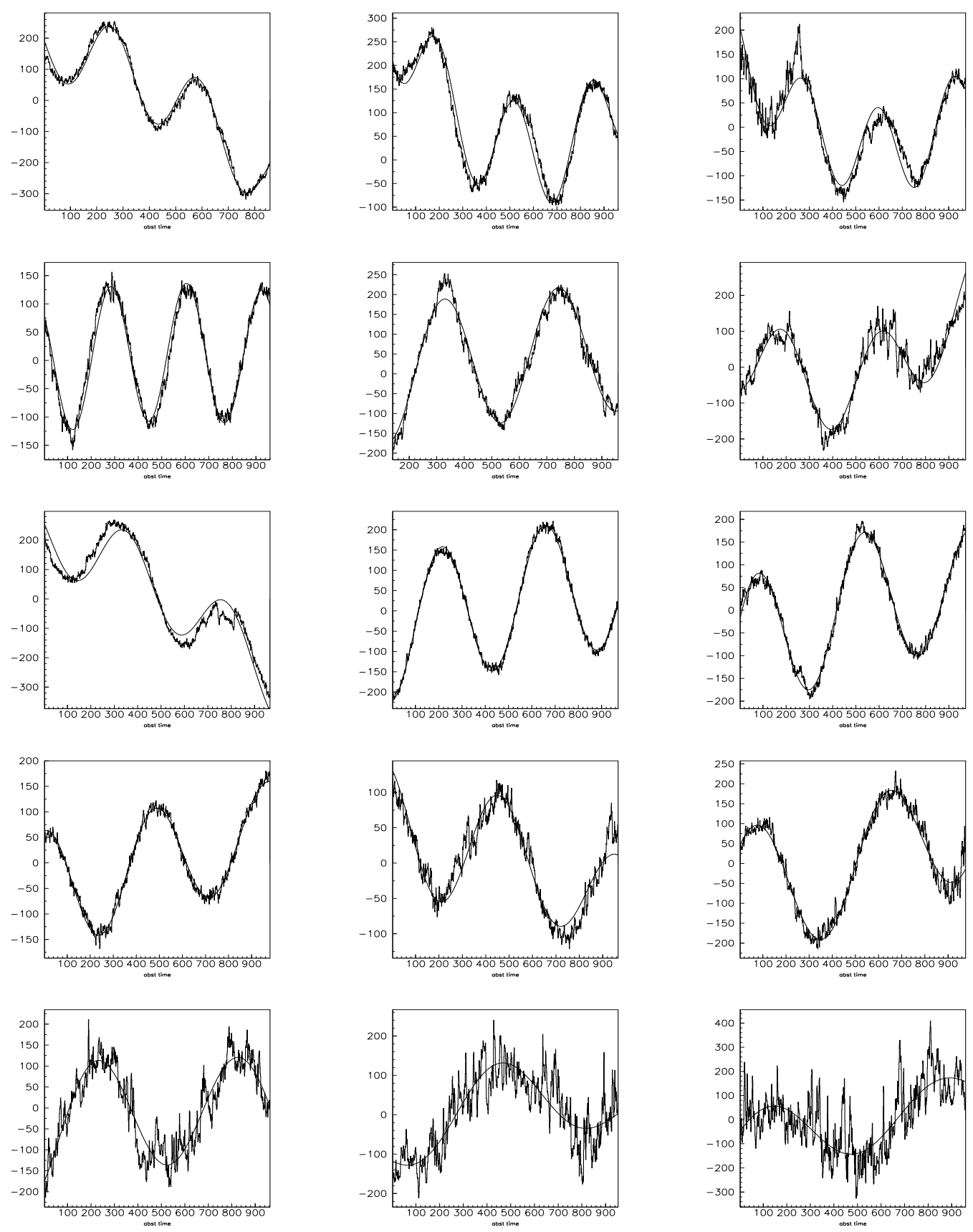

Fig. 6. Result of the global fit to the measurements of the 15 runs (see text).

The data have been compared with the above expression under the assumption that the background term, B, could be parameterized as a quadratic function of time during 
the duration of a given run. In addition to the baseline geometry mentioned earlier, the parameters of relevance include the longitude and latitude of the measuring site, $105^{\circ} 30^{\prime}$ $\mathrm{E}$ and $20^{\circ} 53^{\prime} \mathrm{N}$ respectively, and the declination of the Sun on that day, 23.4 $4^{\circ}$. Fits are performed in two steps; in a first step, run dependent parameters are fine-tuned for each run separately by fixing parameters that are common to all runs; in a second step, a global fit is made to all runs together, fixing the run dependent parameters to their former best fit values and fitting parameters that are common to all runs. The procedure is repeated iteratively and a single iteration is found to be sufficient. Run dependent parameters include three parameters describing a quadratic background and a time off-set allowing for a fine tuning of the start time.

Global parameters include two parameters describing the orientation of the baseline and the amplitude of the solar fringes. The background level that has been fit to each run independently is taken with reference to a slowly varying component illustrated in Figure 5 and described by a form $B_{0}=23+0.0279(t-5.20)^{2}$ where $t$ is the time measured in minutes from noon. It is therefore described as $B=B_{0}+\Delta B$ where $\Delta B$ is a run dependent quadratic function of time. For each individual run, the rms value of $\Delta B$ with respect to its mean has been used as a common uncertainty assigned to each measurement of this particular run. In practice, uncertainties were set to $1.8 \Delta B$ in order to have a $\chi^{2}$ of 1 unit per degree of freedom. The fine tuning of the baseline orientation gave minor shifts with respect to the measured values. The best fit value of the signal is 1439 counts. In order to obtain an estimate of the uncertainty, dominated by the lack of precise knowledge of the background, individual run fits have been repeated, this time fitting not only the four run dependent parameters mentioned earlier but also the amplitude of the signal, the mean and rms values of which are found to be 1483 and 157 counts respectively. The results of the final global fit are shown in Figure 6 for each of the 15 runs. The agreement is excellent.

\section{ESTIMATE OF THE SOLAR FLUX DENSITY}

While the primary aim of the observation was a study of the solar interference fringes, an estimate of the solar flux density has also been obtained. Using the calibration constant of 3.2 counts per $\mu \mathrm{V}$, we obtain the amplitude of the solar signal at the input of the detector, $450 \pm 50 \mu \mathrm{V}$, which, under $50 \Omega$, corresponds to a power of $2.0 \pm 0.5 \mathrm{nW}$. The quoted uncertainty accounts for the lack of precise knowledge of the background, $157 / 1439=11 \%$. Dividing by the system gain $\left(10^{7.6}\right)$, by $\lambda^{2} / 4 \pi\left(0.020 \mathrm{~m}^{2}\right)$, by the system bandwidth $(0.7 \mathrm{MHz})$ and multiplying by 2 (two polarizations) we obtain a flux density of $(7 \pm 2) 10^{-21} \mathrm{~W} / \mathrm{m}^{2} / \mathrm{Hz}$ compared to an average quiet Sun flux of $3.10^{-21} \mathrm{~W} / \mathrm{m}^{2} / \mathrm{Hz}$. A global uncertainty of $\pm 4 \mathrm{~dB}$, attached to the overall gain of the apparatus (including uncertainties on the antenna gain and on the detector calibration constant), must be added to it. In addition, a small correction is necessary to account for the fact that the Sun is not a point source, as implied in Relation 1, but a disk of uniform luminosity with an apparent diameter of 32 arcmin. Integrating Relation 1 over the Sun disk, $S^{2}=E_{1}^{2}\left\{1+r^{2}+2 r \int \cos \left(2 \pi \mathbf{A}_{\mathbf{1}} \mathbf{A}_{\mathbf{2}} \cdot\left(\mathbf{u}_{\mathbf{0}}+\boldsymbol{\varepsilon}\right) / \lambda\right) d \varepsilon^{2} d \alpha /\left(2 \pi \varepsilon_{\max }^{2}\right)\right\}$ where $\mathbf{u}_{\mathbf{0}}$ is the unit vector pointing to the centre of the Sun and $\varepsilon$ is a vector normal to it and having azimuth $\alpha$ around it; the integral runs from 0 to $2 \pi$ in $\alpha$ and from 0 to $\varepsilon_{\max }^{2}=22.10^{-6}$ in $\varepsilon^{2}$. The 
corresponding correction amounts to $15 \%$ on average (i.e. $0.6 \mathrm{~dB}$ ) and has a quadratic dependence on hour angle $H$ of the form $-0.17+3.610^{-4} H+7.510^{-3} H^{2}$.

The present estimate of the flux density of the Sun is therefore $4 \pm 4 \mathrm{~dB}$ above the average emission of the quiet Sun in this frequency range [3]. Indeed, while having long stayed in a quiet state [4], the Sun is now showing signs of entering a new cycle of activity $[5]$.

\section{ACKNOWLEDGEMENTS}

This measurement is a modest attempt at initiating some research in radio astronomy in Vietnam. We hope that it will be followed by many others. We thank warmly those who gave us encouragement, help and support and, in particular, Professor Nguyen Quang Rieu, at the origin of this initiative and Professor Nguyen Van Hieu. We are deeply indebted to Professor Dinh Van Trung for his interest and scientific guidance. We also express our sincere gratitude to Nguyen Phuc, F. Biraud, B. Darchy, Tran Trong My, Tran Minh Van, Nguyen Thi Bao My, who gave us technical help and advice or allowed us to use some of their measuring equipment. A special thank goes to Ms Hoang Mai Anh who welcomed us in her beautiful villa near Hoa Binh. We acknowledge financial support from the Vietnamese Ministry of Science and Technology and from the World Laboratory.

\section{REFERENCES}

[1] Francois Biraud, Observatoire de Paris, Section de Meudon, La Radioastronomie d'Amateur, une Realisation Facile pour Amateur, Astronomie, Novembre 1985, 529.

[2] Nguyen Quang Rieu, Simple Instruments in Radio Astronomy. Astronomy for Developing Countries, IAU Special Session at the 24th General Assembly, 2001, Alan H. Batten, ed.

[3] J. D. Kraus, Radio Astronomy, Mc Graw-Hill Inc, New York, 1966

[4] http://prop.hfradio.org/

[5] http://www.nasa.gov/topics/solarsystem/sunearthsystem/main/News080210-cme.html

Received 29 November 2010. 